\title{
COMPASS results on transverse transverse spin and transverse momentum effects
}

\author{
Anna Martin* \\ University of Trieste and INFN Trieste \\ E-mail: anna.martin@ts.infn.it
}

\section{on behalf of the COMPASS Collaboration}

COMPASS is a fixed target experiment at the CERN SPS with a broad physics programme. An important part of this programme consists in the study of the spin structure of the nucleons by measuring semi-inclusive deep inelastic scattering (SIDIS) of a high energy muon beam off polarised deuteron and proton targets. These measurements allow for a first complete investigation of transverse spin and intrinsic transverse momentum effects. From the data collected in the years 2002, 2003 and 2004 using a transversely polarised ${ }^{6} \mathrm{LiD}$ (polarised deuteron) target, the COMPASS Collaboration has measured the Collins and the Sivers asymmetries [1,2], which allow to access the transversity and the Sivers distribution functions, both particulary interesting in the present theoretical description of the nucleon structure. Using the same data, the other six azimuthal single spin asymmetries expected in SIDIS off transversely polarised targets have been measured [2]. New preliminary results for azimuthal spin asymmetries from the data collected in 2004 with the longitudinally polarised ${ }^{6} \mathrm{LiD}$ target are presented in a different talk at this Workshop [3]. Also, preliminary results for the spin independent azimuthal asymmetries on deuteron have been produced and already shown at several conferences [4]. This talk only addresses the Collins and the Sivers asymmetries. The new results from the data collected in 2007 using a transversely polarised $\mathrm{NH}_{3}$ (polarised proton) target are presented, and the future plans of the COMPASS Collaboration related to the study of transverse spin effects are briefly described.

XVIII International Workshop on Deep-Inelastic Scattering and Related Subjects, DIS 2010 April 19-23, 2010

Firenze, Italy

${ }^{*}$ Speaker. 
Transverse spin and transverse momentum effects at high energy are well established experimentally and are fascinating topics. In order to describe these effects in the QCD framework, the description of the partonic structure of the nucleon has been extended to include the quark transverse spin and intrinsic transverse momentum $\vec{k}_{T}$. In the collinear case, in addition to the rather well known momentum distribution $q(x)$, also indicated with $f_{1}^{q}(x)$, and the helicity distribution $\Delta q(x)$, or $g_{1}^{q}(x)$, a third parton distribution function (PDF) $\Delta_{T} q(x)$ is needed to fully specify the quark structure of the nucleon at the twist-two level. The transversity distributions $\Delta_{T} q(x)$, or $h_{1}^{q}(x)$, gives the probability of having quarks with polarisation parallel or anti-parallel to the transverse polarisation of the parent nucleon, and has only recently been accessed experimentally. When $\vec{k}_{T}$ is taken into account, eight leading twist PDFs are allowed. They are the so-called transverse momentum dependent (TMD) distributions and only three of them survive after integration over the intrinsic transverse momentum, giving $q(x), \Delta q(x)$, and $\Delta_{T} q(x)$. In between these new TMD PDFs, the most famous is the "Sivers function" which describes the correlation between the transverse momentum of quarks in transversely polarised nucleons and the nucleon spin, and which is also very poorly known.

Today, the clearest source of information about the transversity and the Sivers functions are the SIDIS measurements with transversely polarised targets. Results have been produced in the last five years by the HERMES experiment at DESY and, at higher energy, by the COMPASS experiment at CERN. In the case of inclusive hadron production in DIS of longitudinally polarised leptons off transversely polarised targets the cross-section contains eight azimuthal modulations which are harmonic functions of linear combinations of the azimuthal angles of the hadron $\left(\phi_{h}\right)$ and of nucleon spin $\left(\phi_{S}\right)$ defined in the $\gamma^{*} N$ reference system. Among them, those relevant for the extraction of the transversity and the Sivers functions are the amplitudes $\varepsilon_{C}$ and $\varepsilon_{S}$ of the $\sin \Phi_{C}$ and $\sin \Phi_{S}$ modulations, where $\Phi_{C}=\phi_{h}+\phi_{S}-\pi$ and $\Phi_{S}=\phi_{h}-\phi_{S}$ are the "Collins" and "Sivers" angles. From their measurements one obtains the so-called Collins asymmetry $A_{\text {Coll }}=$ $\varepsilon_{C} /\left(D_{N N} \cdot S_{T}\right)$ and the Sivers asymmetry $A_{S i v}=\varepsilon_{S} / S_{T}$. The quantity $D_{N N}=(1-y) /\left(1-y+y^{2} / 2\right)$ is the transverse spin transfer coefficient from the target quark to the struck quark, $y$ is the fractional energy of the virtual photon, and $S_{T}$ is the nucleon polarisation. The Collins asymmetry contains the convolutions of the transversity PDF's and the TMD Collins FF's, which describe the left-right asymmetry in the hadronisation of a transversely polarised quark with respect to the plane defined by its spin and its direction. Informations on the Collins FF's are presently coming from the BELLE Collaboration [5], and these data, together with the SIDIS measurements of the Collins asymmetry allow to extract both the transversity and the Collins functions [6]. The Sivers asymmetry gives access to the convolution of the Sivers function with the known spin independent FF, and thus allows to extract informations on the Sivers function.

The COMPASS Collaboration has measured the Collins and the Sivers asymmetries on deuteron and proton using a $160 \mathrm{GeV} \mu^{+}$beam with a longitudinal polarisation of about $-80 \%$.

The deuteron results for charged and identified hadrons from the data collected with the ${ }^{6} \mathrm{LiD}$ target in the years 2002, 2003, and 2004 have already been published [1]. Both the Collins and the Sivers asymmetries turned out to be compatible with zero, a result which could be explained in terms of cancellation of the $\mathrm{u}$ - and the d-quark contributions in a deuteron target.

In 2007 data were collected using a transversely polarised $\mathrm{NH}_{3}$ target (proton polarisation $\simeq 80 \%$, dilution factor $\simeq 0.15$ ). The analysis of these data for the Collins and the Sivers asymme- 


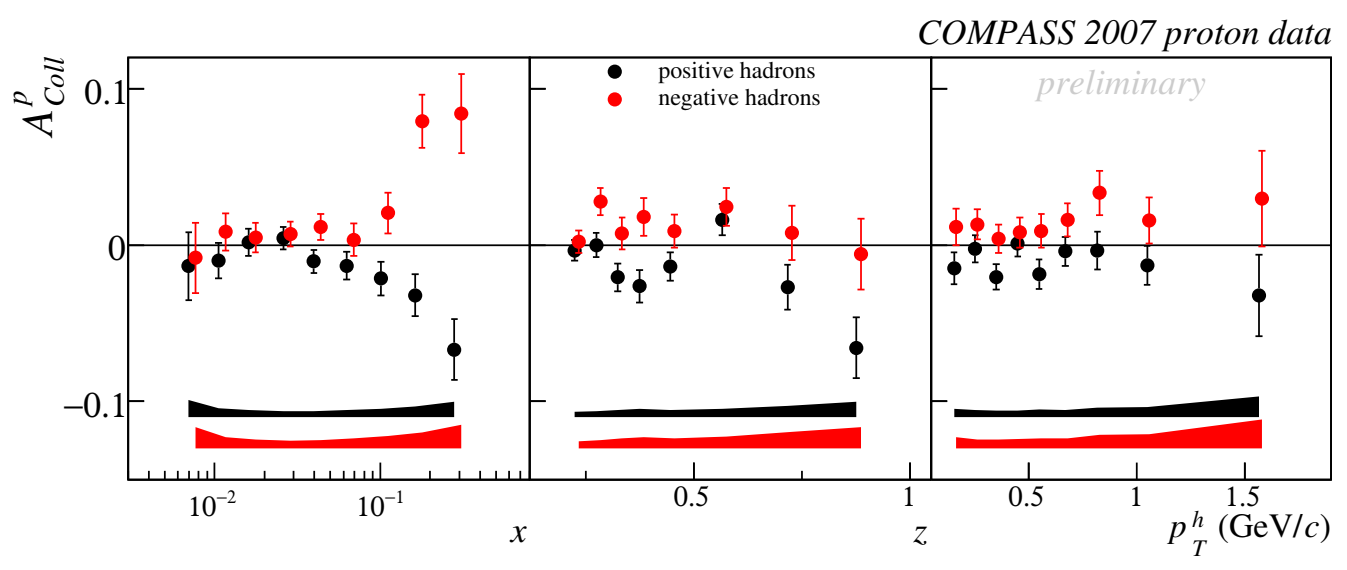

Figure 1: Collins asymmetry on proton as a function of $x, z$ and $p_{T}^{h}$ for positive (black points) and negative (red points) hadrons. The bands give the point to point systematic uncertainty.

tries is now over and the new results shown here have in the mean time been sent for publication [7]. The 2007 experimental apparatus was very similar to the one used in the years 2002-2004. The major changes concern: the new polarised target magnet, with a larger angular acceptance; the target itself, with the material distributed in three cells with opposite polarisation directions in the two outer cells with respect to the central one; the trigger system, in which the electromagnetic calorimeter information was used. As for the deuteron measurements, the data were taken during different periods, each consisting of two sub-periods of 4-5 days, with the reversal of the polarisation direction in all the cells in between. The data quality has been investigated at length looking at the stability of raw and reconstructed data, and performing specific tests on the final SIDIS samples. These tests led to the rejection of part of the data and, in the case of the Sivers asymmetry, of two of the six data taking periods. In the data analysis the DIS events have been selected by asking for a photon virtuality $Q^{2}>1(\mathrm{GeV} / \mathrm{c})^{2}, 0.1<y<0.9$, and a mass of the hadronic final state $W>5 \mathrm{GeV} / c^{2}$. The charged hadrons used in the extraction of the asymmetries were required to have a good momentum reconstruction, a relative energy $z>0.2$, and a transverse momentum with respect to the virtual photon direction $p_{T}^{h}>0.1 \mathrm{GeV} / c$. For each data taking period the asymmetries have been extracted using an extended maximum likelihood method in which all the 8 modulations were included, and binning the data alternatively as function of $x, z$ and $p_{T}^{h}$. Finally, the systematic errors have been evaluated for each asymmetry and each hadron charge independently.

The new results for the Collins asymmetry on proton are shown in fig. 1 as a function of $x$, $z$ and $p_{T}^{h}$ for positive and negative hadrons. They are essentially unchanged with respect to the corresponding preliminary results [8]. As can be seen, at small $x$ the values are compatible with zero both for positive and negative hadrons. In the valence region, at $x>0.1$, where the $Q^{2}$ mean values range from $\sim 7$ to $20(\mathrm{GeV} / \mathrm{c})^{2}$, the Collins asymmetry is definitely different from zero and of opposite sign for positive and negative hadrons. This is an important result since values different from zero at the COMPASS $Q^{2}$ values are a clear indication of the leading twist nature of this asymmetry. Also, the size of the asymmetry, very similar to that measured by HERMES [9] and in agreement with the calculations based on the global fit results [6], shows that the $Q^{2}$ dependence 


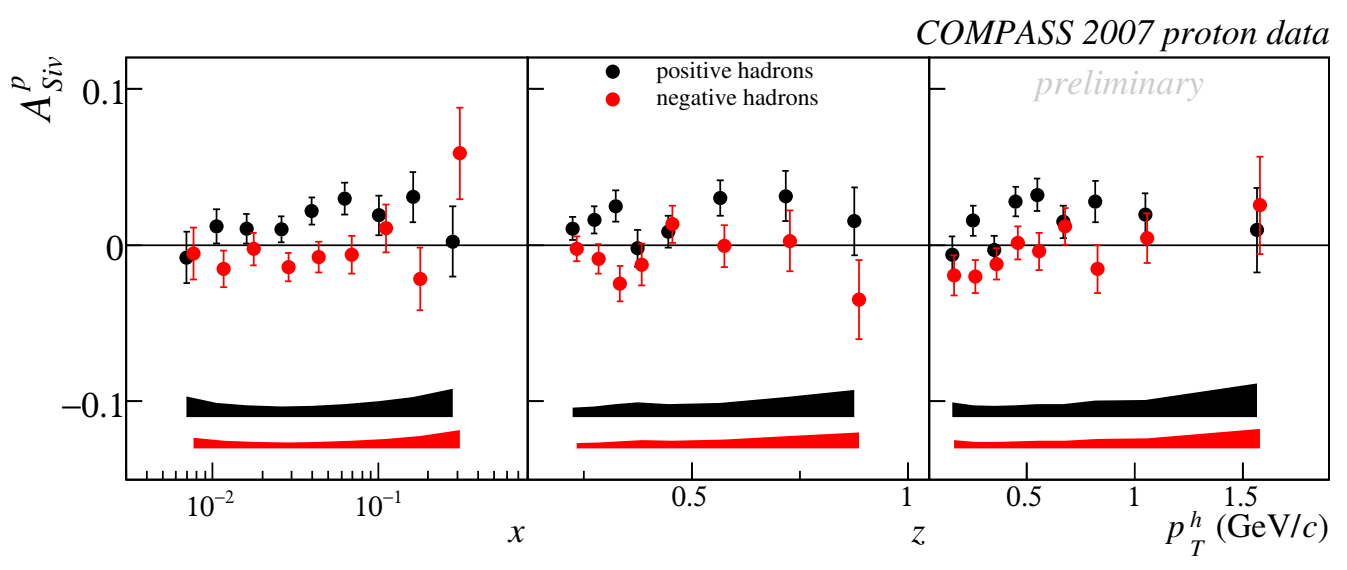

Figure 2: Sivers asymmetry on proton as a function of $x, z$ and $p_{T}^{h}$ for positive (black points) and negative (red points) hadrons. The bands give the point to point systematic uncertainty. For positive hadrons only there is an additional absolute scale uncertainty of 0.01 due to the marginal compatibility between the results from the different periods.

of the Collins FF is not strong, at least in the explored energy range.

For completeness, it must be said that the Collins asymmetry is not the only SIDIS channel proposed to access transversity. The azimuthal asymmetry of the plane containing two oppositely charged hadrons (the "two-hadron asymmetry") is a very promising observable, and COMPASS has already measured quite large asymmetries with the transversely polarised proton target [10]. Although statistically limited, an other interesting channel is the $\Lambda$ and $\bar{\Lambda}$ inclusive production and the most recent COMPASS results are presented in [11].

The first preliminary results for the Sivers asymmetry on proton produced by COMPASS using part of the data collected in 2007 [12] were compatible with zero both for positive and negative hadrons. The new results, which use all the useful statistics, are shown in fig. 2. For negative hadrons the asymmetry is compatible with zero, slightly negative at small $x$, and compatible with the HERMES results [9]. For positive hadrons, positive values are measured over a wide $x$ range. In the overlap region, the asymmetry measured by COMPASS is about a factor of 2 smaller than that observed by HERMES for positive pions and more precise measurements are needed to quantify this difference. A new and more precise measurement will also allow to investigate the $W$ dependence of the Sivers asymmetry, for which hints are given by the present data. As can be seen from fig. 3, where the Sivers asymmetry is shown as a function of $x$ for $W>7.5$ and $W<7.5$ $\mathrm{GeV} / c^{2}$, all the signal seems to be due to the low $W$ region, which also corresponds to lower $Q^{2}$ values. Even if the absolute scale uncertainty of 0.01 acts in the same direction for the two sets of data, the precision of the measurements does not allow to draw definite conclusions on this possible dependence.

Summarising, the COMPASS results for the Collins and the Sivers asymmetries on proton are very interesting and clearly indicate that SIDIS is an appropriate tool to study the properties of the transverse spin and transverse momentum distribution functions. New high energy measurements, complementary to the results coming from the JLab experiments, are needed to better investigate 


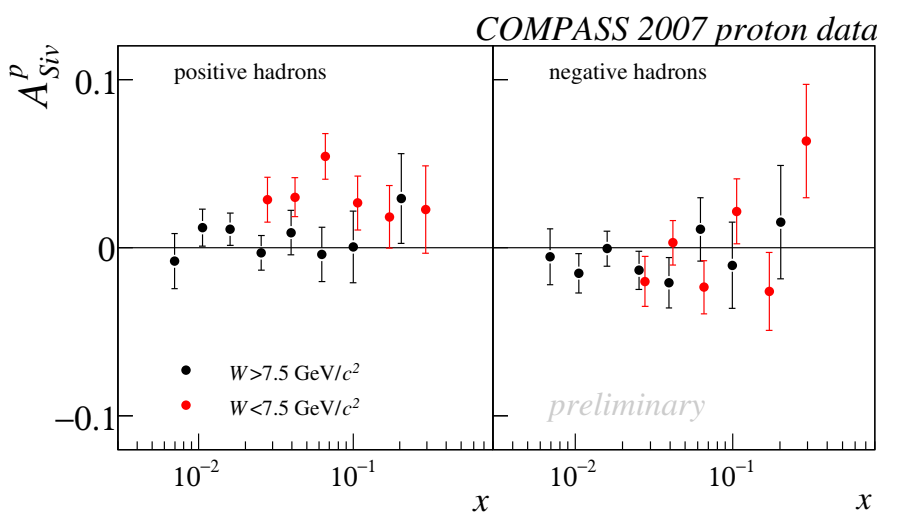

Figure 3: The Sivers asymmetry on proton for positive (left) and negative (right) hadrons as a function of $x$. The red (black) points give the values for $W<7.5(>7.5) \mathrm{GeV} / c^{2}$.

the properties of the transversity distribution and to clarify the properties of the Sivers asymmetry. In 2010 the COMPASS Collaboration will take data with the transversely polarised $\mathrm{NH}_{3}$ target and it should be possible to improve considerably the precision of the present results. In the near future, COMPASS is planning to further contribute to the study of transverse spin and transverse momentum effects by measuring Drell-Yan processes with transversely polarised targets [13], a very interesting and challenging channel complementary to SIDIS.

\section{References}

[1] V. Y. Alexakhin et al. [COMPASS Collaboration], Phys. Rev. Lett. 94 (2005) 202002; E. S. Ageev et al. [COMPASS Collaboration], Nucl. Phys. B 765 (2007) 31; M. Alekseev et al. [COMPASS Collaboration], Phys. Lett. B 673 (2009) 127.

[2] A. Kotzinian [COMPASS collaboration], DIS2007 Proceedings, arXiv:0705.2402 [hep-ex].

[3] I. Savin [COMPASS collaboration], these Proceedings.

[4] A. Bressan [COMPASS Collaboration], DIS2009 Proceedings, arXiv:0907.5511 [hep-ex].

[5] A. Vossen [BELLE Collaboration], these proceedings.

[6] M. Anselmino et al., Nucl. Phys. Proc. Suppl. 191 (2009) 98; S. Melis et al., these proceedings.

[7] M. G. Alekseev et al. [COMPASS Collaboration], CERN-PH-EP-2010-013, arXiv:1005.5609.

[8] A. Bressan [COMPASS Collaboration], arXiv:0907.5508 [hep-ex].

[9] G. Schnell [HERMES Collaboration], these Proceedings, and references therein.

[10] H. Wollny [COMPASS Collaboration], DIS2009 Proceedings, arXiv:0907.0961 [hep-ex].

[11] D. Kang [COMPASS Collaboration], these Proceedings.

[12] S. Levorato [COMPASS Collaboration], Transversity 2008 Proceedings, arXiv:0808.0086 [hep-ex].

[13] O. Denissov [COMPASS Collaboration], these Proceedings. 